\title{
ALAIN PERRONNET \\ Problèmes soulevés par la programmation de la méthode des éléments finis
}

Mémoires de la S. M. F., tome 49-50 (1977), p. 145-158

<http://www.numdam.org/item?id=MSMF_1977_49-50_145_0>

(C) Mémoires de la S. M. F., 1977, tous droits réservés.

L'accès aux archives de la revue « Mémoires de la S. M. F. » (http://smf. emath.fr/Publications/Memoires/Presentation.html) implique l'accord avec les conditions générales d'utilisation (http://www.numdam.org/conditions). Toute utilisation commerciale ou impression systématique est constitutive d'une infraction pénale. Toute copie ou impression de ce fichier doit contenir la présente mention de copyright.

\section{Numdam}


Calculateurs en Math. (1975 - LIMOGES)

Bu11. Soc. math. France

Mémoi re 49-50, 1977, p. 145-158

PROBLEMES SOULEVES

PAR LA PROGRAMMATION DE LA METHOIE

DES ELEMENTS FINIS

par Alain PERRONNET

Le but de ces journées étant l'utilisation des calculateurs en mathématiques, nous nous bornons ici à énoncer les problèmes rencontrés lors de 1'implémentation en souhaitant que les programmes issus de cette recherche puissent profiter au plus grand nombre.

1 - La méthode des éléments finis découverte dans les années 60 connaît actuellement un essor considérable à tous les niveaux des Sciences de l'ingénieur. Cette méthode est présentée ici sur un problème simple après, avoir dégagé une formulation mathématique abstraite du phénomène physique, mécanique ...

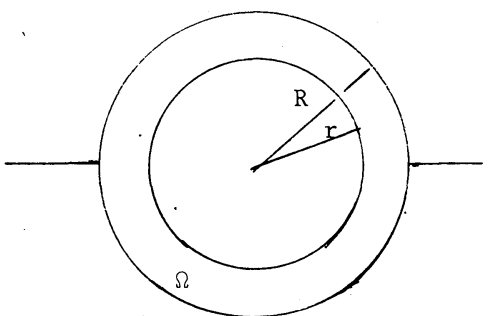

Soit un tube contenant du sodium liquide à haute température $U_{1}$, plongé dans deux fluides de température $\mathrm{U}_{2}, \mathrm{U}_{3}$. La température $u$ en chaque point de $\Omega=\left\{(\mathrm{x}, \mathrm{y}), \mathrm{r}^{2}<\mathrm{x}^{2}+\mathrm{y}^{2}<\mathrm{R}^{2}\right\}$ est sous certaines hypothèses physiques solution de $\Delta \mathrm{u}=0$ dans $\Omega$.

(1)

$$
\begin{gathered}
u=U_{1} \operatorname{sur} \Gamma_{1}=\left\{(x, y), x^{2}+y^{2}=r^{2}\right\} \\
-\lambda \frac{\partial u}{\partial n}=h_{j}\left(u-U_{j}\right) \text { sur } \Gamma_{j} j=2,3 \quad \Gamma_{2}=\left\{(x, y) / x^{2}+y^{2}=R^{2} y>0\right\} \\
\Gamma_{3}=\left\{(x, y) / x^{2}+y^{2}=R^{2} y<0\right\} \\
\lambda, h_{j} \geqslant 0
\end{gathered}
$$

ou sous une forme équivalente

$$
\begin{aligned}
& \left\{\lambda \int_{\Omega} \frac{\partial u}{\partial x} \frac{\partial v}{\partial x}+\frac{\partial u}{\partial y} \frac{\partial v}{\partial y} d x d y+\sum_{j=2}^{3} h_{j} \int_{\Gamma_{j}} u v d \Gamma=\sum_{j=2}^{3} h_{j} \int_{\Gamma_{j}} U_{j} v d \Gamma\right. \\
& \mathrm{u} \in \mathrm{K}^{\mathrm{U}^{1}}=\left\{\mathrm{w} \in \mathrm{H}^{\mathrm{I}}(\Omega), \mathrm{w}_{\mid} \Gamma_{1}=\mathrm{U}_{1}\right\} \\
& \text { pour tout } v \in \mathrm{K}^{\circ}
\end{aligned}
$$

Plus généralement : Trouver

$$
\left\{\begin{array}{l}
u \in \mathrm{K}^{U}=\left\{\mathrm{w} / \mathrm{w} \in \mathrm{V}, \mathrm{v}=\mathrm{U} \text { sur } \Gamma_{1}\right\} \quad \text { solution de } \\
\mathrm{a}(\mathrm{u}, \mathrm{v})=(\mathrm{f}, \mathrm{v}) \quad \forall \mathrm{v} \in \mathrm{K}^{\circ}
\end{array}\right.
$$

avec . $\mathrm{V}$ espace de Hilbert de norme \|\|

- a( , ) forme bilinéaire continue sur. $V$ vérifiant 


$$
a(v, v) \geqslant \alpha\|v\|^{2} \text { pour tout } v \in K^{\circ}
$$

- $f$ une forme linéaire continue sur $V$

Le théorème de Lax Milgram nous assure l'existence et l'unicité de u.

\section{2 - Définitions - Le problème approché -}

\section{Soient :}

- un recouvrement de $\bar{\Omega}$ par des segments, triangles, quadrangles, tétraèdres pentaèdres ou hexaèdres suivant le cas. (Dans le cadre de notre exemple $\bar{\Omega}=\mathrm{U} T$ avec des triangles);

- $P$ un espace de polynômes

$$
\text { - } V_{h}=\left\{v / v \in c^{\circ}(\bar{\Omega}),\left.v\right|_{T} \in P\right\} \cdot
$$

Si P désigne les polynômes du ler degré à deux variables, p $\epsilon$ P est caractérisé par ses valeurs aux trois sommets du triangle $\mathrm{T}$.

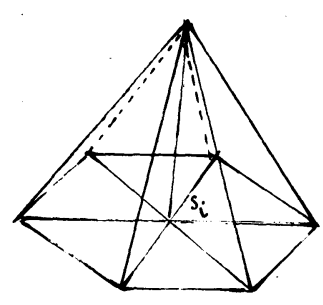

$$
\text { Si } \mathcal{f}=\left\{s_{j} / j \in J\right\} \text { représente les }
$$
sommets du maillage, nous définissons pour $s_{i} \in \mathcal{S}, \delta_{i j}$ symbole de Kronecker, $w_{i} \in V_{h}$ par $w_{i}\left(S_{j}\right)=\delta_{i j} \quad \forall j \in J$ ce qui entraîne :

support $\left(w_{i}\right)=$ réunion des triangles de sommets $\mathrm{S}_{i}$.

Les $w_{i}$ forment une base de $V_{h}$ et posons

$v \in K_{h}^{U}=\left\{w / w \in V_{h}, w\left(S_{j}\right)=U_{1}\left(S_{j}\right)\right.$ pour tout $\left.s_{j} \in \Gamma_{1}\right\}$

$$
\text { i.e } \quad v(x, y)=\sum_{i \in J-I} v\left(S_{i}\right) \cdot w_{i}(x, y)+\sum_{j \in I} U_{1}\left(S_{i}\right) w_{i}(x, y)
$$

Le problème approché s'énonce :

Trouver $u_{h} \in K_{h}^{U}$ solution de

i.e

$$
a\left(u_{h}, v_{h}\right)=\left(f, v_{h}\right) \quad \forall v_{h} \in K_{h}^{\circ}
$$

$\sum_{i \in J-I} u_{h}\left(s_{i}\right) a\left(w_{i}, w_{j}\right)=\left(f, w_{j}\right)-\sum_{i \in I} U_{1}\left(s_{i}\right) a\left(w_{i}, w_{j}\right) \forall j \in J-I$

comme $w_{i}$ est connu explicitement sur $T$, nous avons

$\left.\sum_{T}\left(\sum_{i \in J-I} u_{h}\left(S_{i}\right) a_{T}\left(w_{i}, w_{j}\right)\right)=\sum_{T}\left(f, w_{i}\right)_{T}-\sum_{i \in I} U_{1}\left(S_{i}\right) a_{T}\left(w_{i}, w_{j}\right)\right) \quad \forall j \in J-I$

1 'indice. $T$ indiquant 1 a réstriction à $T$.

La matrice du système $A x=b$ avec

$$
\begin{aligned}
x=\left(\sum_{T} a_{T}\left(w_{i}, w_{j}\right)\right)_{i, j \in J-I} & \left(u_{h}\left(s_{i}\right)\right){ }_{i \in J-I} \\
b & =\left(\left(f, w_{j}\right)-\sum_{i \in I} U_{1}\left(s_{i}\right) a\left(w_{i}, w_{j}\right)\right){ }_{j \in J-I}
\end{aligned}
$$

est creuse 
$\sum_{T} a_{T}\left(w_{i}, w_{j}\right)=0$ dès que $i$ et $j n^{\prime}$ appartiennent pas à un même élément.

3 - La difficulté mathématique du problème- réside dans :

- La recherche de la formulation continue la plus sujette à l'approximation

- La construction des espaces de dimension finie $V_{h}, K_{h}^{U}$ liés aux contraintes contenues dans $\mathrm{V}$; par exemple div $\vec{u}=0$ pour un fluide incompressible.

- La démonstration de la convergence de $u_{h}$ vers $u$ lorsque le recouvrement s'affine, et d'une majoration de $u_{h}-u$ dans un certain espace et pour une certaine norme (cf. Ciarlet - Riavart - Thomas et les bibliographies associées).

- Stabilité des algorithmes de résolution du problème approché (propagation des erreurs d'arrondis, vitesse de convergence ...)

Ces problèmes ne sont pas abordés ici bien que constituant la presque totalité du travail mathématique. Lorsque toutes ces difficultés sont résolues la programmation requiert le reste de notre attention.

\section{4 - Quels sont les programmes à écrire?}

4.1. Décomposition du domaine en éléments -

La dimension de $1^{\prime}$ espace $\mathbb{R}, \mathbb{R}^{2}, \mathbb{R}^{3}$ contenant $\Omega$ rend croissante 1 a difficulté.

Si dans $\mathbb{R}$ ou $\mathbb{R}^{2}$, les algorithmes programmés sont satisfaisants il en va tout autrement dans le cadre tridimensionnel !

Exemple - Recouvrir $\Omega=\left\{x, y, z / x^{2}+y^{2}+z^{2}>R^{2}\right.$

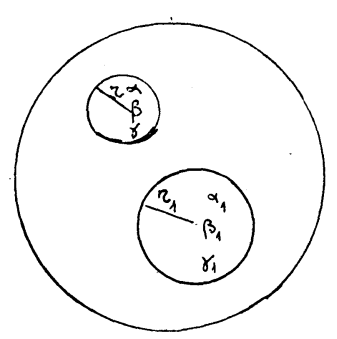

$$
\begin{aligned}
& \left\{\begin{array}{l}
(x-\alpha)^{2}+(y-\beta)^{2}+(z-\gamma)^{2}>r^{2} \\
\left(x-\alpha_{1}\right)^{2}+\left(y-\beta_{1}\right)^{2}+\left(z-\gamma_{1}\right)^{2}>\Omega_{1}^{2}
\end{array}\right. \\
& \text { avec } \\
& \left(\alpha-\alpha_{1}\right)^{2}+\left(\beta-\beta_{1}\right)^{2}+\left(\gamma-\gamma_{1}\right)^{2} \geqslant\left(r+r_{1}\right)^{2} \\
& \alpha^{2}+\beta^{2}+\gamma^{2}<(R-r)^{2} \\
& \alpha_{1}^{2}+\beta_{1}^{2}+\gamma_{1}^{2}<\left(R-r_{1}\right)^{2}
\end{aligned}
$$

par des tétraèdres (qui ne dégénèrent pas !) n'est pa's facile. Pour $\Omega \subset \mathbb{R}^{2}$, nous disposons de quatre algorithmes programmés

- décomposition semi-automatique -

Chaque "gros " élément du recouvrement défini par l'utilisateur est subdivisé en $\mathrm{N}^{2}$ sous-élément, les sommets générés étant éventuellement projetés sur une courbe (figure 1). 

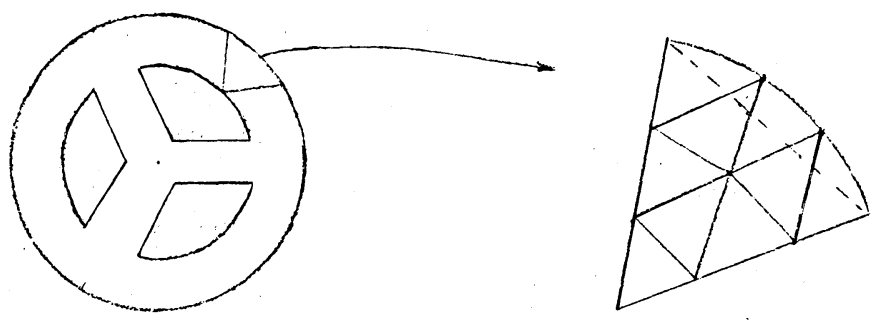

- décomposition automatique -

La donnée des sommets sur la frontière du domaine suffit à faire engendrer le recouvrement à partir de la comparaison des angles intérieurs au domaine formé par trois d'entre eux consécutifs (figure 2).
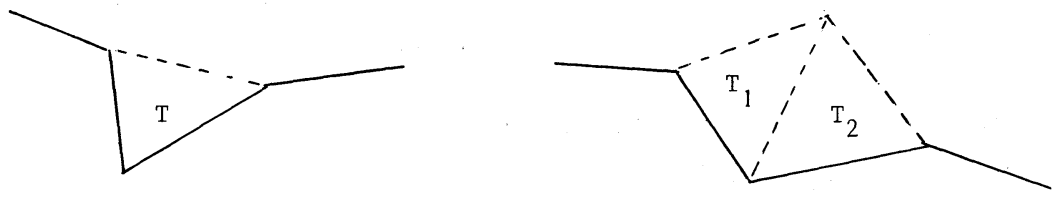

- affinage local de la décomposition - (figure 3)

- recollement par symétries, rotations de décomposition préalablement calculées (pour un recouvrement de la première figure celui du $1 / 6$ du domaine suffit).

4.2. La précision des résultats est majorée par une fonction du type $\mathrm{h}^{\mathrm{m}} / \rho^{\mathrm{n}}$ $\underline{\mathrm{m}>\mathrm{n}}$

h diamètre de l'élément

$\rho$ valeur mesurant l'écrasement de l'élément; par exemple diamètre de la sphère inscrite pour le tétraèdre.

Par suite, la forme des éléments doit être vérifiée, voire modifiée s'ils dégénèrent.

Des programmes de visualisation du maillage

- sur traceur de courbes

- console graphique conversationnelle

furent développés pour faciliter les retouches des triangles et quadrangles.

Si $\Omega \subset \mathbb{R}^{3}$, la visualisation n'est pas toujours très claire car la "profondeur" est difficile à juger sur une feuille de papier ou sur un écran.

4.3. Le plus grand soin doitt être mis à la formation - résolution du système qui peut devenir gigantesque (plusieurs milliers ou dizaines de milliers d'équations). La mémoire centrale de l'ordinateur le plus puissant ne'suffit plus. Les algorithmes déterminent la gestion des fichiers sur disques ou bandes magnétiques (accès séquentiel ou direct). 
Les méthodes d'inversion du système sont directes ou itératives. Les premières sont généralement préférées pour leur coût et la possibilité d'estimer le temps nécessaire au calcul.

Si la matrice A est définie positive, symétrique, 1a méthode de Cholesky avec stockage du profil est employée. E1le part de la remarque :

la partie de chaque colonne comprise entre le premier terme non nul et le terme diagonal de la partie triangulaire supérieure est nécessaire et suffisante pour calculer $U$ matrice triangulaire supérieure telle que $A={ }^{t} U . U$.

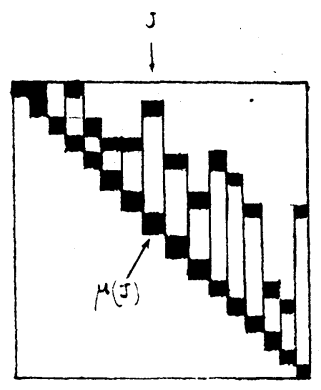

En numérotant ces coefficients de haut en bas et de la gauche vers la droite, en stockant $\mu(\mathrm{I})=$ numéro du $I^{\text {ème }}$ coefficient diagonal alors 1 'adresse de $A(I, J)$ est $\mu(\mathrm{J})-\mathrm{J}+\mathrm{I}$.

Pour un problème important (plus de mille équations) la mémoire centrale insuffisante nous oblige à regrouper les segments utiles par blocs, de taille telle que deux blocs puissent y prendre place. Une succession d'entrées-sorties mémoire centrale $\leftarrow \rightarrow$ mémoire auxiliaire permet le calcul de la solution.

Remarquons que le nombre de coefficients de $\mathrm{U}$ dépend de la numérotation des sommets la longueur du Jème segment étant égale dans le cas de notre exemple à $\mathrm{J}-\mathrm{I}+1$ où $\mathrm{I}$ est le plus petit numéro de sommet d'un triangle contenant aussi $\mathrm{S}_{\mathrm{J}}$. Il convient donc de renuméroter les sommets de telle sorte que l'un d'entre eux numéroté, ses voisins (sommets de triangles le contenant) soient à leur tour numérotés le plus vite possible. Le problème de trouver la ou les permutations qui réalisent le min $\sum_{J} S_{i}, S_{j} \in T$ max $j+i+1$ n'est pas résolu. Les algorithmes $j \geqslant i$

actuels sont heuristiques.

La méthode frontale (méthode de GAUSS couplée à 1'élimination des inconnues associées au sommet $j$ dès que celui-ci n'appartient plus aux autres éléments avec la matrice du système formée élément par élément et non pas ligne par ligne) évite le stockage complet de la matrice. A chaque instant ne sont en mémoire centrale que les lignes associées aux sommets des éléments déjà traités et réapparaissant par la suite.

Exemple :

Les triangles (1) (2) (3) déjà traités, le sommet 2 n'apparait plus ensuite, est par conséquent éliminé. Remarquons qu'à ce stade sont en mémoire les lignes 


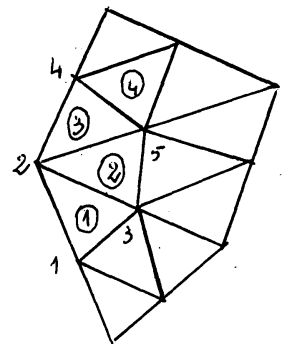

$1,3,5,4$ et que les coefficients de celles-ci ne sont pas définitifs. Le triangle (4) n'ayant pas par exemple apporté sa contribution aux coefficients associés aux sommets 4, 5. La ligne éliminée est stockée sur un disque et une lecture arrière du fichier permet de trouver la solution.

Au cours du calcul le front des sommets actifs (en mémoire centrale) se déplace L'encombrement mémoire dépend alors de ce cheminement c'est-à-dire de la numérotation des éléments.

Les algorithmes de numérotation des sommets engendrent une bonne rénumérotation des éléments. Il suffit de renuméroter les éléments non encore renumérotés dès que 1'un de leurs sommets l'est.

Le problème général de trouver pour tout recouvrement, $1 \mathrm{a}$ ou les numérotations optimales des éléments afin de minimiser le nombre de sommets actifs n'est pas résolu.

Remarquons que si la programmation de la méthode frontale dépend directement du recouvrement celle de CHOLESKY peut être utilisée en "boite noire" dès que la partie triangulaire supérieure de A, le pointeur $\mu$ et les seconds membres sont stockés convenablement.

La méthode des super-éléments. (méthode frontale partielle sur des groupes d'éléments) permet de s'affranchir de la limite due à la taille du front. Elle sera programmée prochainement.

4.4. L'exposé vient d'être fait avec des éléments triangulaires sur lesquels la solution est polynomiale de degré 1. Ce ne sont pas les seuls. Des interpolations plus fines faisant intervenir les valeurs des dérivées premières, secondes, sont parfois nécessaires (éléments de coque). Si la frontière est courbe, la précision gagnée par 1'emploi de polŷnomes de degré plus élevé se trouve dégradée par 1'approximation de la frontière courbe par la corde.

Une technique d'élément courbe permet de

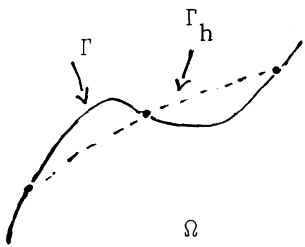
récupérer la bonne précision. Par exemple si les polynômes sont de degré 2, le domaine d'intégration approché sera limité par la parabole passant par les sommets et le "milieu" de l'arc. L'intégration numérique des expressions devient alors obligatoire. 
La difficulté du calcul des $a_{T}\left(w_{i}, w_{j}\right)$ et $\left(f, w_{j}\right)_{T}$ va alors croissante, tout comme la démonstration de'la convergence de $u_{h}$ vers $u$ et de sa majoration $\left(\| u_{h}-u||\right)$.

4.5: La méthode des résidus permet de vérifier la valeur des résultatş

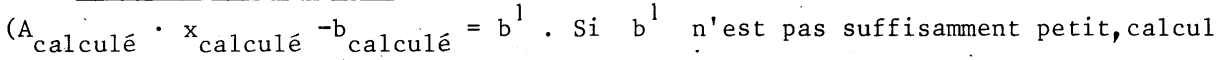
de $A x^{1}-b^{1}=b^{2}, \ldots$ )

4.6. L'exploitation de $\mathrm{n}$ feuilles de 1isting est longue et fastidieuse. Des programmes de visualisation'des résultats sont alors les bienvenus.

Trois types d'algorithmes travaillant sur $\Omega \subset \mathbb{R}^{2}$ sont actuellement programmés 1 - déformée d'une pièce de $\mathbb{R}^{2}$ c'est-à-dire perturbation du domaine initial $\Omega$ (figure 4).

2 - tracé des isovaleurs dans $\mathbb{R}^{2}$

$3-z=u(x, y)$ visualisé par des sections à $x=c^{\text {te }}$ avec élimination des lignes cachées (figure 5).$$
y=c^{t e}
$$

De nouveau dans $\mathbb{R}^{3}$, le problème est beaucoup plus difficile et demandera de passer par des coupes du domaine.

5. Dès qu'un ensemble de programmes à vocation générale devient volumineux, un certain nombre de désirs apparaissent :

- possibilité de changer des algorithmes sans tout réécrire (modularité)

- vu les temps calculs et les possibilités d'erreurs, permettre les reprises en évitant de recommencer les calculs au début.

Une manière de concilier ces deux aspects réside dans le passage des paramètres entre algorithmes par l'intermédiaire des fichiers.

Pour changer un algorithme ou module il suffit alors de respecter la structure du ou des fichiers d'entrée et de sortie. En contrepartie ces derniers doivent être définis très généralement afin d'englober tous les cas actuels et futurs.

- soulager l'utilisateur de l'apprentissage des codes de perforations, rédaction du programme d'appel dans un langage d'informatique, gestion des fichiers, des tableaux ...

Comme la totalité de nos programmes sont en FORTRAN pour des raisons d'universalité, un pré-processeur a'interprétation en FORTRAN de macro instructions s'avère utile afin de

- rapprocher le langage des données de celui de l'ingénieur ...

- détecter les erreurs

- décharger de toute gestion 1'utilisateur (temps calcul, dimensionnement des tableaux, programme principal d'enchainement, gestion des fichiers). 
Pour éviter l'emploi de l'assembleur (spécifique de chaque machine) deux 'exécutions s'avèrent nécessaires :

1. Interprétation des données et macro instructions conduisant à gênérer le programme principal comprenant

- le dimensionnement d'un tableau égal au maximum de ceux utilisés dans chaque module (cf. structure modulaire), (avec certaines contraintes au niveau de 1 'écriture des modules).

- Les instructions de mise à jour d'un fichier de gestion contenant l'état d'exécution des modules et fichiers

- déclaration des fichiers avec leur méthode d'accès

- définition de la structure d'OVERLAY

- le recouvrement éventuel des fichiers au fur et à mesure de 1'exécution (même étiquette passée en paramètre).

2. Exécution proprement dite du programme issu de 1. et comprenant

- compilation

- édition de lien avec les modules stockés sous forme de modules de chargement non exécutables

- chargement - exécution.

Passer d'une machine à une autre conduit seulement à

- évaluer le rapport des vitesses d'exécution

- réécrire les formats décrivant les cartes contrôle

Un tel préprocesseur est en cours d'étude.

Le cadre de l'étude précédente était limité aux problèmes elliptiques linéaires En fait la méthode des éléments finis peut être appliquée avec bonheur sur les problèmes

- elliptiques non linéaires

- paraboliques 1inéaires ou non

- hyperboliques linéaires ou non

pour lesquels la totalité ou partie des modules explicités précédemment peuvent

être rééemployés.

Par contre de nouveaux algorithmes de

- résolution de systèmes non linéaires

- intégration pas à pas

- calculs de valeurs propres

doivent être développés. 
ARGYRIS J.H. et son équipe.- An automatic system for kinematic analysis.University of Stuttgart.- I.S.D. report (1971)

CIARLET-RAVIART. - General Lagrange and Hermite interpolation in $\mathrm{R}^{\mathrm{n}}$ with applications to finite element method.- Arch. Rat. Mech. Anal.- 46 (1972) p. $177-189$.

CIARLET-RAVIART. - Interpolation theory over curved elements with applications to finite element methods. - Computer methods in applied mechanics and engineering. - p. 217-250.- vol. $\mathrm{n}^{\circ}$ 2.- (August 1972).

EGELAND 0. - ARALDSEN P.0: - SESAM 69.- A general purpose finite element method program.- Computers and Structure.- vo1. 4.- p. 41-68.- Pergámon press (1974).

FUCHS - ROY - SCHREM. - Hyper matrix solution of large sets of symmetric positive definite linear equations.- p. 197-216.- Computer method in applied mechanics and engineering.- vol. 2 (1972) (cf. la bibliographie)

GEORGE A.- Computer implementation of the finite element method.- Stanford (february 1971).

JOLY - KOUTCHMY - LEGENDRE - PERRONNET. - Le programme MEFISTO. - à paraître au Laboratoire d'Analyse Numérique.- Université PARIS VI.

MAC NEAL R.H.- Some organizational aspects of NASTRAN.- Nuclear engineering and design 29 (1974).- p. 254-265.- North Holland Publishing company.

SUTHERLAND - SPROULL - SCHUMECKER. - A characterization of ten Hidden-surface algorithmes.- Computing Surveys.- vol. 6.- $\mathrm{n}^{\circ} 1$ (march 1974).

THOMAS.- Méthode des éléments finis équilibres. Méthodes des éléments finis hybrides duaux pour les problèmes elliptiques du second degré.- Laboratoire d'Analyse numérique.-- Université PARIS VI (1975).

VOUILLON.- TITUS : Entreprise SPIE BATIGNOLLES.- CREUSOT-LOIRE.

WILLIAMSON.- Hidden line plotting.- Communication of the A.C.M.- (february (1972) vol. 15, $\mathrm{n}^{\circ} 2$

ZIENKIEWICZ.- La méthode des éléments finis.- Ediscience (Paris 1973).

\author{
A. PERRONNET \\ Laboratoire d'Analyse Numérique \\ Université PARIS VI, tour 55-65 \\ 4, Place Jussieu \\ 75230 PARIS Cédex 05
}




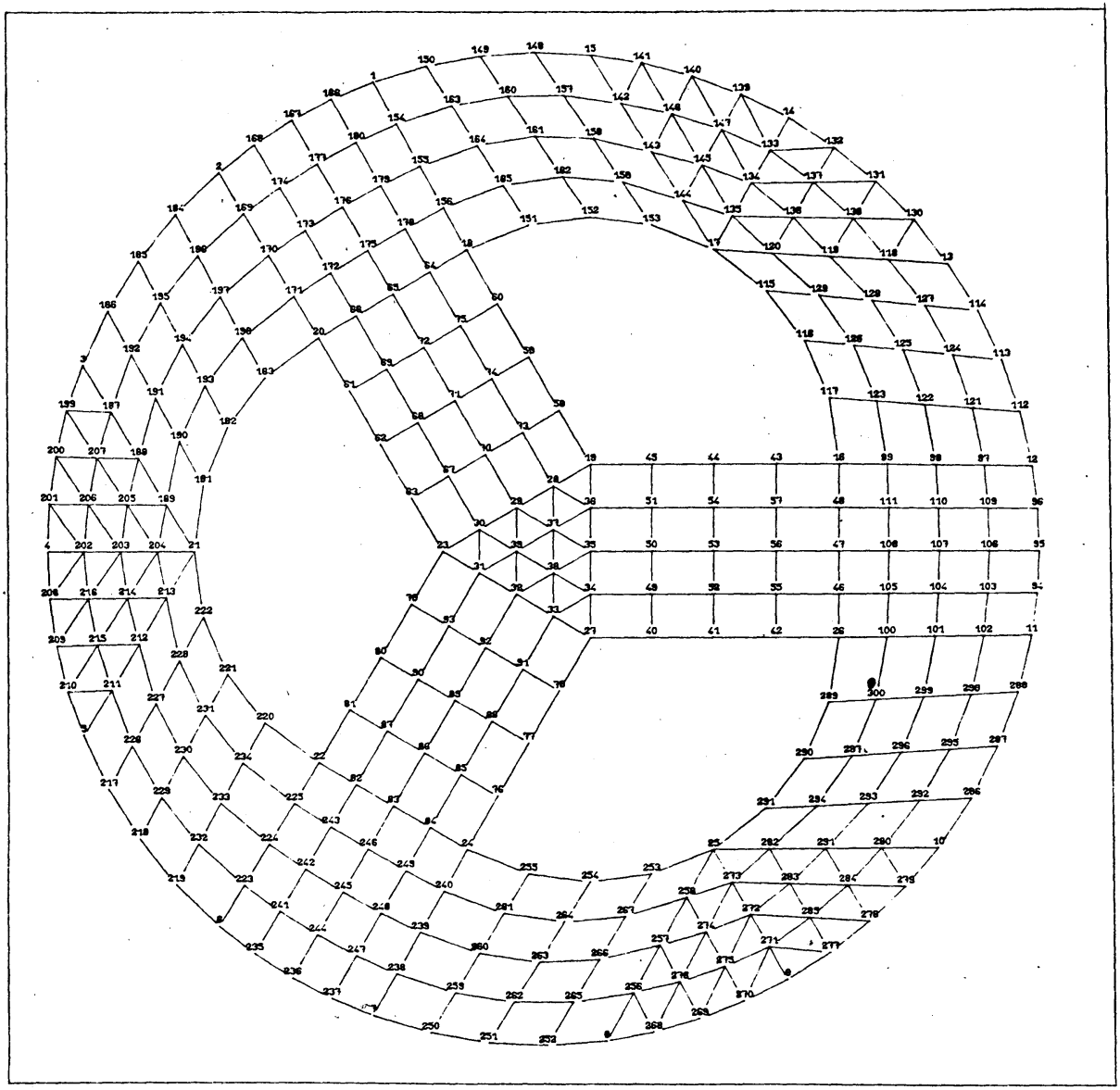

Figure 1 


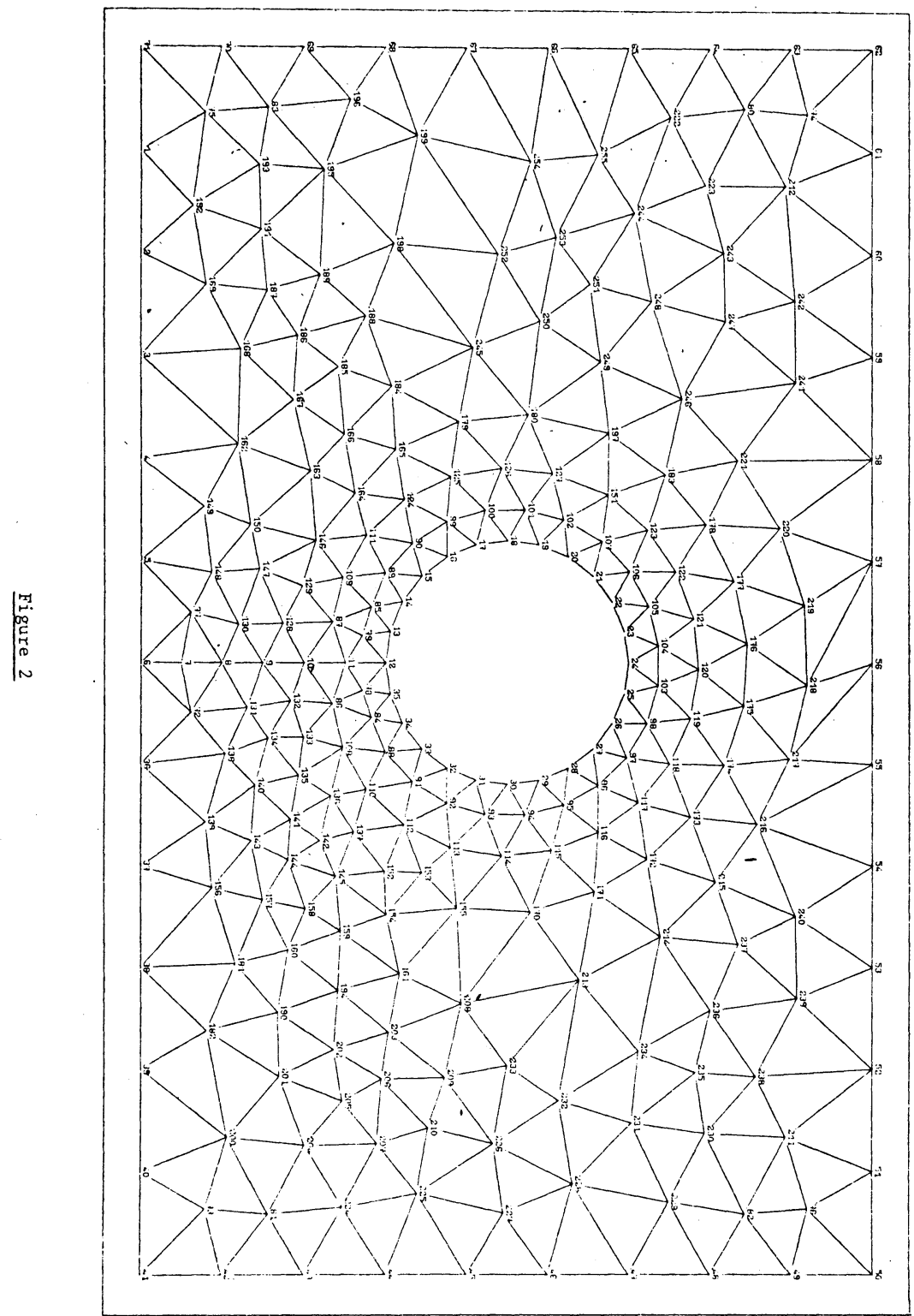




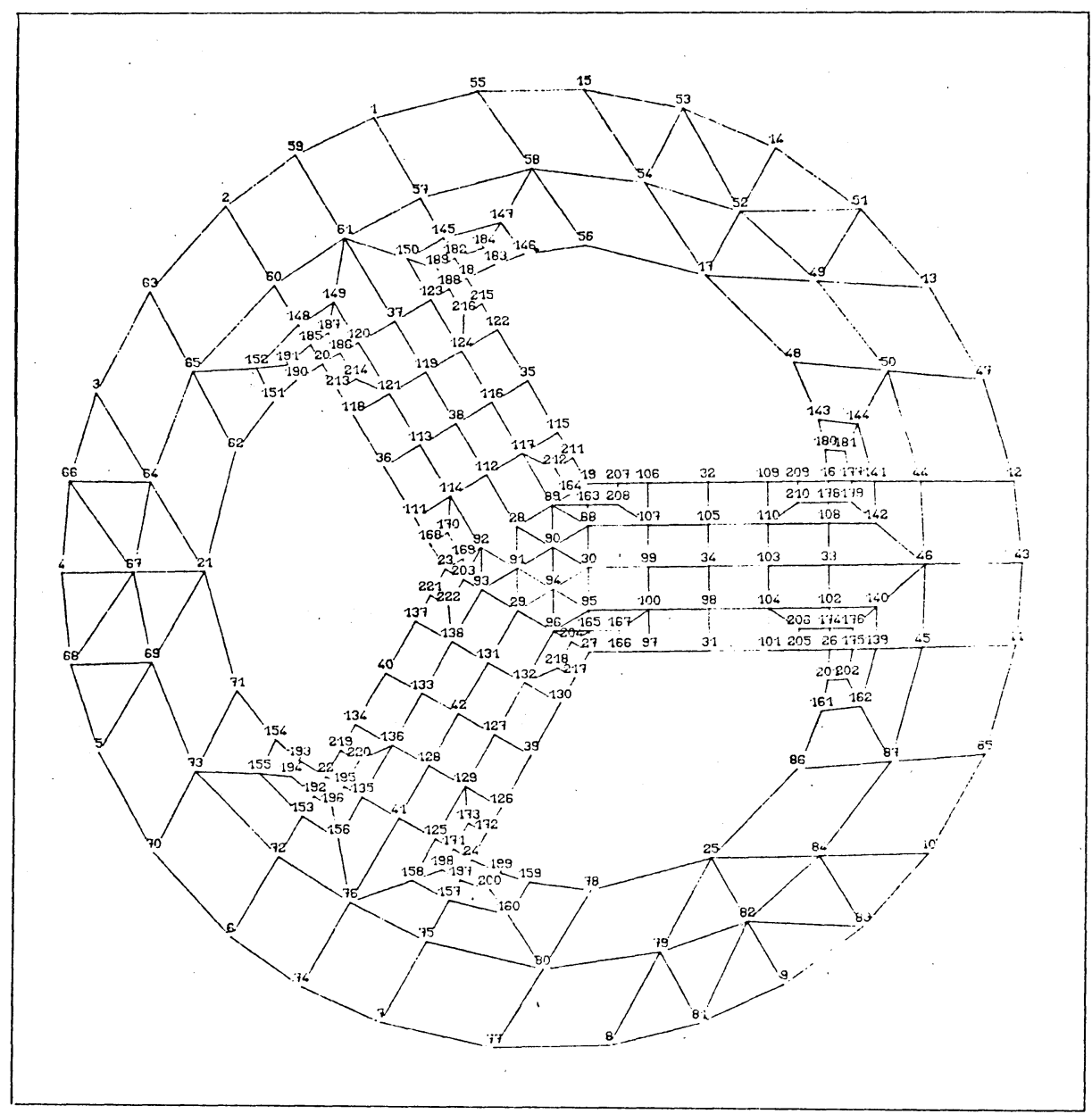

Figure 3 


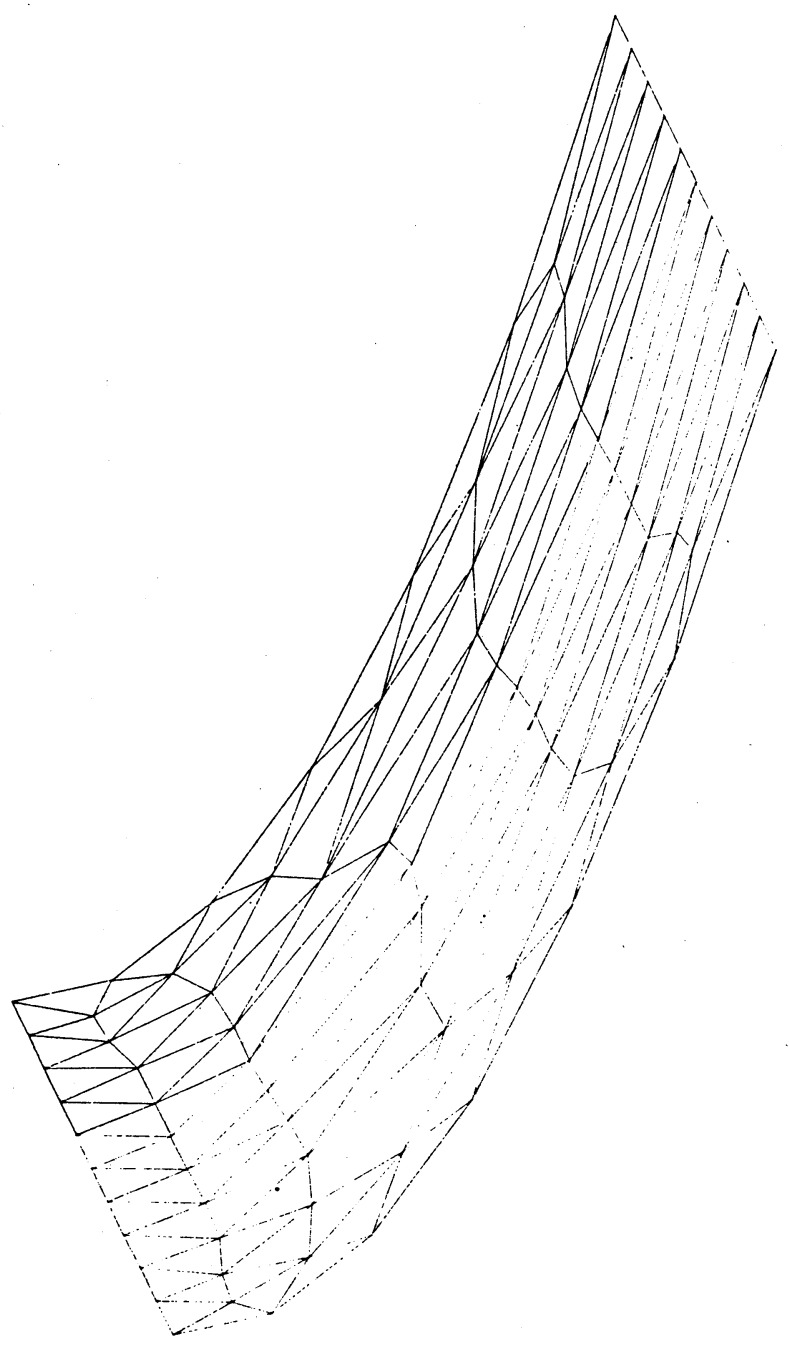

Figure 4 


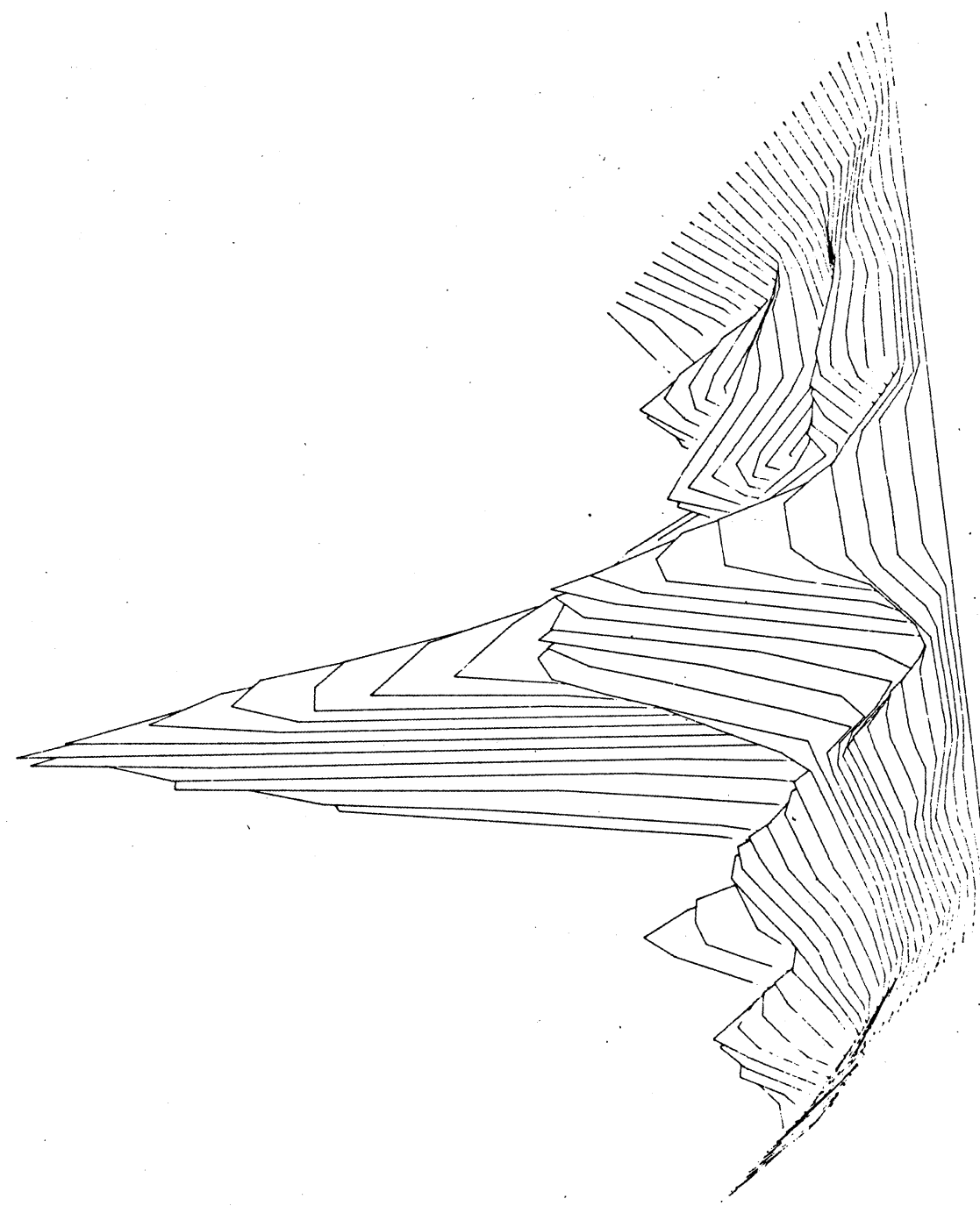

